\title{
Lenalidomide in Relapsed and Refractory Multiple Myeloma
}

\section{Dattatreya Mukherjee, MBBS Student, Medical Researcher, Jinan University, P.R China}

\begin{abstract}
:
Improvement in the treatment options in multiple myeloma(MM) makes the survival rate into double during recent years but most of the patient eventually relapses. At the time of relapses, the use of alternative drugs is the current practice. Lenalidomide is an immuno-modulators and it is one of the most versatile drug which is used in several Hematological disease treatment including MM. Several clinical trials have shown a better progression free survival(PFS) and overall survival(OS) with Lenalidomide based regimens. In Pollux study, the regimen with Daratuzumab with Lenalidomide and dexamethasone has shown much better OS and PFS than only Lenalidomide and Dexamethasone. But Some times patients are refractive to Lenalidomides. In that case Pomalidomide or bortezomib based regimen is used. Although Lenalidomide is one of the most promising drug to treat both first line and Relapse-refractory case of MM. Hemato oncology is a challenging topic and its correlated with personalized medicine this days. Different clinical trial are going on with different regimen. In some patient some regimen is showing good result and in some other regimen is giving good prognosis. So its always tough to tell which is the best regimen. In this article we will focus on use of Lenalidomide based regimes and we will see the median OS and median PFS of all these Lenalidomide based regimen. Althrough there is a good room for research on Len refractive cases and personalized treatment in multiple myeloma in both front line and relapsed and refractory cases.
\end{abstract}

Key Words: Lenalidomide, Relapse, refractory, Multiple Myeloma.

\section{Introduction:}

MM is characterized by the excess amount of monoclonal plasma cell in the bone marrow associated with monoclonal protein in blood and urine. Around 50 years ago the use of combined melphalan and prednisone had shown an extension of median of patients with MM by 2-3 years.(1)

In the year 2006, The International Myeloma Working Group (IMWG), has modified the definition of relapse and refractory MM and relapsed MM is defined as "relapse in patient who must have achieved at least minimal response(MR) which either become non responsive while on salvage therapy or progresses within 60 days of last treatment".(2,3) Primary refractive myeloma defines as disease that is non responsive, patient who have never achieved a MR or better with any therapy. $(2,3)$

Lenalidomide is the most potent derivative of thalidomide that has been found to be less toxic and more active than its older analogue. Lenalidomide is mostly used in multiple myeloma in both frontline and relapsed-refractory cases. Apart from this Lenalidomide is a great drug in $5 \mathrm{q}$ deleted myelodisplastic syndrome(MDS). Compound formula of lenalidomide is $\mathrm{C} 13 \mathrm{H} 13 \mathrm{~N} 3 \mathrm{O} 3$ with a molar mass of $259.261 \mathrm{~g} / \mathrm{mol}$. Single agent Lenalidomide was effective and well tolerated 
in both phase 1 and phase 2 study was response rates ranging from $29 \%$ to $39 \%$ in patients with RRMM who had received 3 prior therapeutic regimen.(4). In 2005, FDA gave approval to Lenalidomide to treat Haematological malignancies. Lenalidomide gives a great result in the treatment of MM and MDS.

Thalidomide was the parent drug which came to reduce nausea and vomiting in pregnancy. But In 1960, a wonder happened all the mother who took Thalidomide gave birth to limb less baby. So Thalidomide is teratogenic and its band from the market. Although in 90s thalidomide was used to treat multiple myeloma with Melphalan.But all the drugs of this group like thalidomide, Lenalidomide and Pomalidomide are not recommended during pregnancy.

\section{Treatment of RRMM with Lenalidomide:}

The biochemical relapse is defined as the significant amount of increase in M protein. Amount of $\mathrm{M}$ protein will be more than equal to $10 \mathrm{mg} / \mathrm{L}$ or in urine $500 \mathrm{mg} / 24$ hours. Clinical relapse is defined as CRAB( hypercalcemia, Renal Failure, Anemia, Bone deformity) and increase in plasmacytomas. It can also be defined as increase in free light chain or FLC of more than $200 \mathrm{mg} / \mathrm{L}$ in a 2 months interval test.

Treatment selection is very important by fitness scores. Palumbo A etal.(6) descibes a scoring system of 0-5 on which the treatment, dosing is dependent on the scope. 0 means unfit patient and its tough to treat this patient. 5 means its great to take the drug with its written dose. So Dose and regimen depends on the fitness score.

A paper of Neils WC published in 2011 has described that Lenalidomide treatment is the best choice in RRMM with associated to neuropathy.

Most promising Lenalidomide based regimen for treating RRMM

1. Lenalidomide plus dexamethasone

2. Elotuzumab with Lenalidomide and dexamethasone

3. Ixazomib with Lenalidomide and dexamethasone

4. Daratuzumab with Lenalidomide and dexamethasone

5. Carfilzomib with Lenalidomide and dexamethasone

Pollux(6) study is done on Daratuzumab-Lenalidomide-Dexamethasone (research group) to Lenalidomide-dexamethasone (controlled group). $n=569$. The overall survival rate (ORR) is $92.9 \%$ for research group and $76.4 \%$ to controlled group and complete response (CR) was $51.2 \%$ and $21 \%$ respectively. OS HR is 0.63 . 


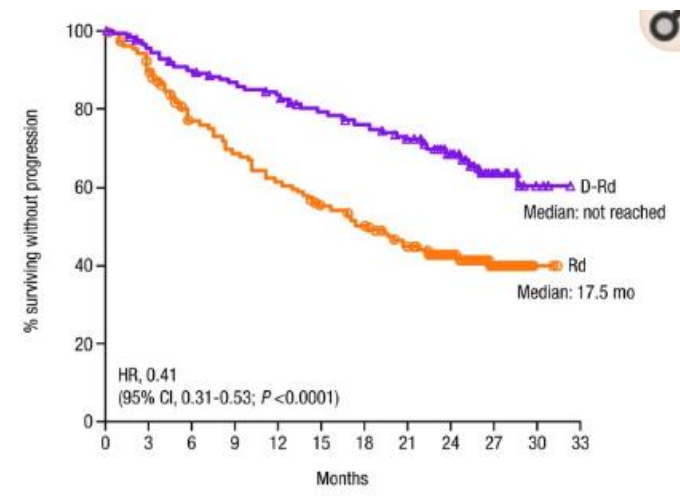

Fig 1. Survival free progression in pollux study. (6)

In Aspire Trial (7) was done on Carfilzomib- Lenalidomide-Dexamethasone (research Group) with Lenalidomide -dexamethasone (Control Group). Median OS is 48.3 months to 40.4 months respectively. The patient who got one prior line therapy, they have 11 months to 6 months of OS respectively. Adverse effects like acute renal failure, cardiac failure, ischemic health disease and thrombocytopenia is seen. OS HR is 0.73 .

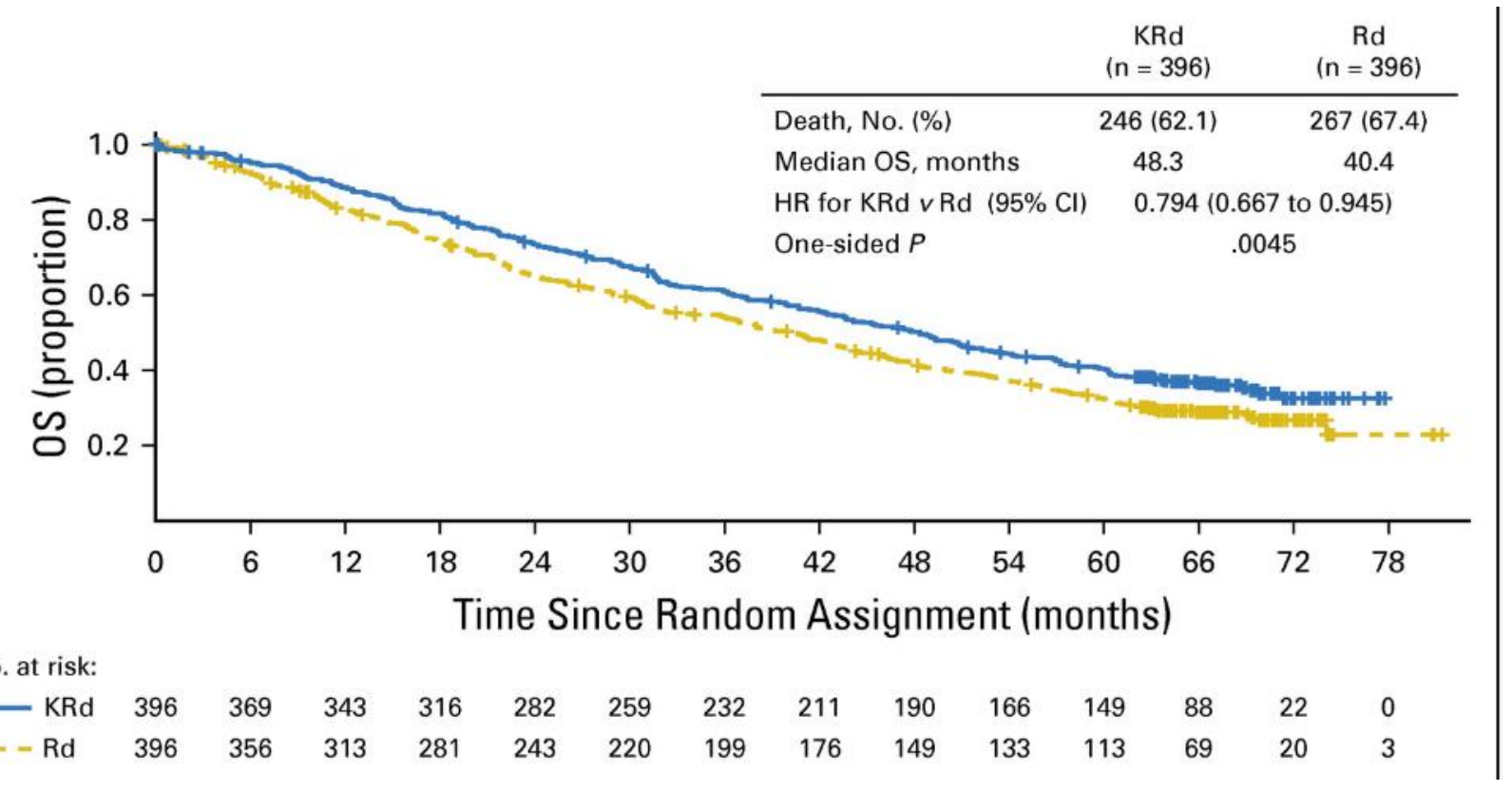

Fig 2: OS of Aspire Study. (7)

In Tourmaline MM1 (8) study ixazomib-Lenalidomide-Dexamethsone to Plecebo group. The research group has shown better OS and PFS than Plecebo group.

Elotuzumab- Lenalidomide-Dexamethasone is giving to patient over Lenalidomidedexamethasone. The research regimen is showing higher OS and PFS than the control regimen. Only Adverse effect is grade 3 Haematological disorder like Lymphocytopenis, Thrombocytopenia and anemia.(9) OS HR is 0.78. 
In some clinical trials has gone where patient has treated with Lenalidomide (LEN) but the patient eventually relapsed and then a different combination tried like in CASTOR trial (10) Daratuzumab- Bortezomib- Dexamethsone tried over Bortezomib and Dexamethsone. It's a phase 3 trial on 498 patients who have previously treated with Len. The one year PFS is $60.7 \%$ respectively over $26.9 \%$. The ORR is also better in research group. Hematologic Adverse effects are the most important concerns. Thrombocytopenia, anemia, neutropenia is common adverse effects seen.

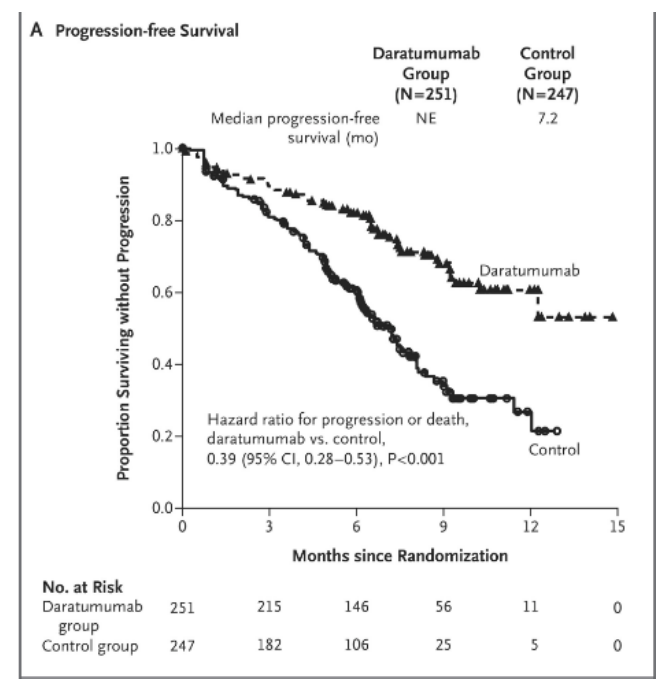

PFS of CASTOR study. (10)

Another Trial named Optimism (11) where Pomalidomide-Bortezomib-Dexamethasone is used over Bortezomib-Dexamethasone in 559 patients who have treated with LEN in frontline therapy. The PFS is 11,2 months over 7.1 months in control group. Most common adverse effect was neutropenia and infections.

Candor Study(12) shows Carfilzomib-daratuzumab-Dexamethasone over Carfilzimubdexamethasone on 466 patients who were treated before on bortezomib or lenalinomide in the front line. The research group shows much better prognosis than control group. This is a ongoing study.

Another study Panaroma study is going on and within few years the result will be received.

So all the new regimens are showing better result than the old result. The triple regimen is giving always a better result in PFS, ORR, OS. But adverse effect in triple regimen is also higher. So to treat these patients a good set up is needed. Most common in triple regimen in anemia or thrombocytopenia. So a blood bank is needed to treat. There are lots of studies going on with different regimen of drugs. With this Stem cell transplantation is also important. Monoclonal antibodies are great in the treatment of MM. Apart from this immunotherapy, CAR therapies are also coming. So in next 30 years the treatment of MM will be more developed and more personalized. 


\section{Lenalidomide refractory cases:}

Lenalidomide refractory cases are the case when patient is resistant to Lenalidomide. Then physician gives different drug regimen. Pomalidomide is the new Immunomodulators. In this case physician use bortezomib, carfizomib, pomalidomide based regimen with low dose dexamethasone. For example: Bortezomib with low dose Dexamethasone or Pomalidomide Daratuzumab and low dose dexamethasone.

The real challenge comes in India when patient is refractive to both Lenalidomide and Bortezomib. In that case the regimen like Pomalidomide-dexamethasone, Pomalidomidecarfilzomib-dexamethasone or monoclonal antibody based regimen is used. These regimens are new, still now researches are going on so drugs are also much costly. Daratuzumab is showing a great result now days.

To see the Lenalidomide refractive cases Len refractive patients are treated with Pomalidomide and dexamethasone or bortezomib and dexamethasone. These patients have showing a good result. First len was given in 5-15 mg and $25 \mathrm{mg}$ but in that case results are also not promising in case of overall survival. Duration of Len treatment is also not very important in LEN refractory cases. So physicians treat with different regimens in LEN refractory patients.

According to the papers, the Endeavor, Castor, Optimisim and Candor study most of patients have relepsed after LEN therapy or they are LEN refractive.

Now in LEN refractory cases Monoclonal antibodies are used like the combination of Daratuzumabs. In a trial Isatuximab is using in combinations of pomalidomide- low dose dexamethasone overpomalidomide and low dose dexamethasone.(13) Most of the patients are LEN refractory and research group shows much better PFS and OS than control group. Median PFS is 11.5 months over 6.4 months.

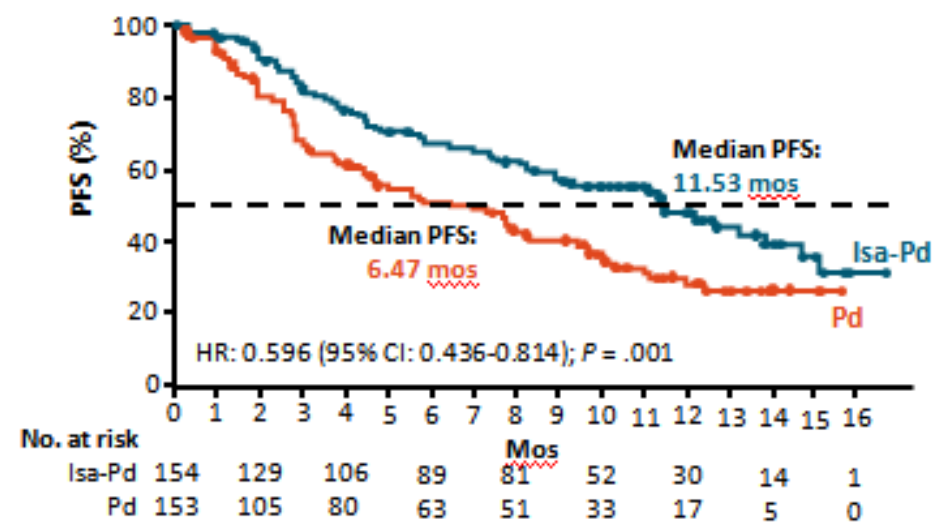

PFS of Isa-Pd over Pd (13)

Another Phase 2 study is Eloquent II study where elotuzumab-pomalidomide-dexamethasone is used over pomalidomide-dexamethsone in LEN refractory parients. The research group shows PFS of 10.3 months over 4.7 months in control group. (14) 


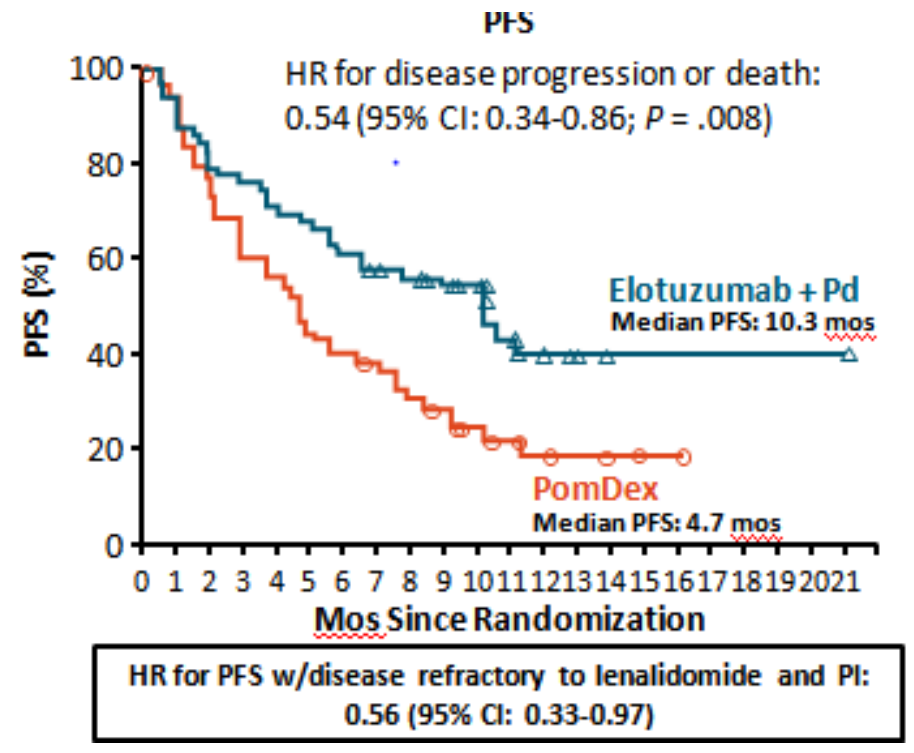

PFS of the study. (14)

A very new trial is going on LEN refractory cases that is IKEMA phase 3 trial (NCT03275285) with Isatuxumab-carfilzomib-dexamethasone tp carfilzomib-dexamethasone. Still now the full rest didn't came of this trial although this showing a good PFS than the control group.

Still now a lots research is pending on LEN refractory cases. A various combinations are going on to treat this cases. Still now it's not clear why refractivity happens. So there are a great room to work on LEN refractory and cytogenetics relapse. In many cases LEN is a good drug in mentenance therapy.

So in future more regimen will be tried with monoclonal antibodies, CAR therapy or immune therapy.

Personalized therapy is trying to understand much more molecular level. So in next 30 years these refractive cases can be treated easily and patient can show great PFS, OS, CR.

\section{Adverse effect of Lenalidomide (Medscape)}

Adverse effects are the side effects. Lenalidomidemide has less adverse effects. It doesn't show very serious adverse effects. Thalidomide shows severe side effects and both the drugs are not recommended during pregnancy. The main adverse effects are given bellow.

Thrombocytopenia(62\%), Neutropenia(59\%), diarrhoea(48\%), prioritus(42\%) Nausea(35\%) Rash (35\%)etc.

This are the adverse effect in sigle lenalidomide therapy, in regimen the most common adverse effect is thrombocytopenia, anemia, lymphopenia. Sometimes ischemic heart disease, neuralgia also occurs. 


\section{Conclusion:}

Lenalidomide is one of the most promising drug in RRMM treatment. It's a second gen immunomodulators which shows much better PFS and OS. Although some patients shows Lenalidomide refractivity. For those mostly pomalidomides or Bortezomib based regimen is given.

The triple therapy is showing great result. Low dose dexamethasone is used as high dose dexamethasone regimen doesn't show a better PFS or OS.

Lenalidomide is a old drug but still now its one of the most important drug. Hematologists says it's a versatile drug, because it shows some miracle in result. MDS and MM both are very critical disease with a short PFS, OS. But lenalidomise makes a significant increase in those. 5q del. MDS is greatly treated by Lenalidomide now day.

Lots of researches are going on with the relapse and refractory MM, So in future we will have more precise molecule, immune based treatment and personalized treatment.

\section{*Main paper: Mukherjee, Kalyan \& Mukherjee, Dattatreya. (2020). Last 10 years Review on Therapeutic strategies on Relapsed and Refractory Multiple Myeloma. 10.13140/RG.2.2.21991.88486.}

\section{Reference}

1.Anderson, Kenneth C.(2012) "The 39th David A. Karnofsky Lecture: bench-to-bedside translation of targeted therapies in multiple myeloma." Journal of clinical oncology : official journal of the American Society of Clinical Oncology vol. 30,4; 445-52. doi:10.1200/JCO.2011.37.891

2. Durie BG, Harousseau JL, Miguel JS, et al. International uniform response criteria for multiple myeloma [published correction appears in Leukemia. 2006 Dec;20(12):2220] [published correction appears in Leukemia. 2007 May;21(5):1134]. Leukemia. 2006;20(9):1467-1473.

doi:10.1038/sj.leu.2404284

3. Anderson KC, Kyle RA, Rajkumar SV, et al. Clinically relevant end points and new drug approvals for myeloma. Leukemia. 2008;22(2):231-239. doi:10.1038/sj.leu.2405016 
4. Silvia Gentili, Sagar Lonial. (2014) Relapsed and Refractory Multiple Myeloma: New Therapeutic Strategies, Hematology/Oncology Clinics of North America,28 (5), p861-890. doi.org/10.1016/j.hoc.2014.06.008

5. Palumbo A, Bringhen S, Mateos MV, et al. Geriatric assessment predicts survival and toxicities in elderly myeloma patients: an International Myeloma Working Group report [published correction appears in Blood. 2016 Mar 3;127(9):1213] [published correction appears in Blood. 2016 Mar 3;127(9):1213] [published correction appears in Blood. 2016 Aug 18;128(7):1020]. Blood. 2015;125(13):2068-2074. doi:10.1182/blood-2014-12-615187

6. Dimopoulos MA, San-Miguel J, Belch A, et al. Daratumumab plus lenalidomide and dexamethasone versus lenalidomide and dexamethasone in relapsed or refractory multiple myeloma: updated analysis of POLLUX. Haematologica. 2018;103(12):2088-2096. doi:10.3324/haematol.2018.194282

7. Siegel DS, Dimopoulos MA, Ludwig $\mathrm{H}$, et al. Improvement in Overall Survival With Carfilzomib, Lenalidomide, and Dexamethasone in Patients With Relapsed or Refractory Multiple Myeloma. J Clin Oncol. 2018;36(8):728-734. doi:10.1200/JCO.2017.76.5032

8. Mateos MV, Masszi T, Grzasko N, et al. Impact of prior therapy on the efficacy and safety of oral ixazomib-lenalidomide-dexamethasone vs. placebo-lenalidomide-dexamethasone in patients with relapsed/refractory multiple myeloma in TOURMALINE-MM1. Haematologica. 2017;102(10):1767-1775. doi:10.3324/haematol.2017.170118

9. Lamb YN. Elotuzumab: A Review in Relapsed and/or Refractory Multiple Myeloma. Drugs. 2018;78(14):1481-1488. doi:10.1007/s40265-018-0969-4

10. Palumbo A, Chanan-Khan A, Weisel K, et al. Daratumumab, Bortezomib, and Dexamethasone for Multiple Myeloma. N Engl J Med. 2016;375(8):754-766. doi:10.1056/NEJMoa1606038

11. Richardson PG, Oriol A, Beksac M, et al. Pomalidomide, bortezomib, and dexamethasone for patients with relapsed or refractory multiple myeloma previously treated with lenalidomide (OPTIMISMM): a randomised, open-label, phase 3 trial. Lancet Oncol. 2019;20(6):781-794. doi:10.1016/S1470-2045(19)30152-4

12. Dimopoulos, Meletios; Quach, Hang; Mateos, Maria-Victoria; Landgren, Ola; Leleu, Xavier; Siegel, David; Weisel, Katja; Yang, Hui; Klippel, Zandra; ZahltenKumeli, Anita; Usmani, Saad. Carfilzomib, dexamethasone, and daratumumab versus carfilzomib and dexamethasone for patients with relapsed or refractory multiple myeloma (CANDOR): results from a randomised, multicentre, openlabel, phase 3 study. The Lancet, ISSN: 1474-547X, Vol: 396, Issue: 10245, Page: 186-197. 10.1016/s0140-6736(20)30734-0

13. Attal M, Richardson PG, Rajkumar SV, et al. Isatuximab plus pomalidomide and low-dose dexamethasone versus pomalidomide and low-dose dexamethasone in patients with relapsed 
and refractory multiple myeloma (ICARIA-MM): a randomised, multicentre, open-label, phase 3 study [published correction appears in Lancet. 2019 Dec 7;394(10214):2072]. Lancet.

2019;394(10214):2096-2107. doi:10.1016/S0140-6736(19)32556-5

14. Dimopoulos, Meletios \& Dytfeld, Dominik \& Grosicki, Sebastian \& Moreau, Philippe \& Takezako, Naoki \& Hori, Mitsuo \& Leleu, Xavier \& LeBlanc, Richard \& Suzuki, Kenshi \& Raab, Marc \& Richardson, Paul \& McKiver, Mihaela \& Jou, Ying-Ming \& Shelat, Suresh \& Robbins, Michael \& Rafferty, Brian \& San-Miguel, Jesús. (2018). Elotuzumab plus Pomalidomide and Dexamethasone for Multiple Myeloma. The New England journal of medicine. 379. 1811-1822. 10.1056/NEJMoa1805762. 